\title{
Craniectomía descompresiva en encefalitis herpética. Caso clínico
}

\author{
RAÚl BUSTOS B. ${ }^{1}$, SEBASTIÁN VIGUERAS A. ${ }^{2}$, JAIME PINTO V. ${ }^{2}$ \\ 1. Medicina Intensiva Pediatrica, UCI Pediátrica Hospital Guillermo Grant Benavente Concepción. \\ 2 Neurocirujano, Servicio de Neurocirugía Hospital Guillermo Grant Benavente Concepción Chile.
}

\begin{abstract}
Decompressive craniectomy in herpes simplex encephalitis. Clinical case

Introduction: The morbidity and mortality of herpes simplex encephalitis (HSE) have decreased with the use of acyclovir. However, some patients develop focal hemorrhagic necrosis and edema in the temporal lobe, with a subsequent elevation of intracranial pressure. Clinical cases: We report the clinical outcomes of two children with HSE who developed severe intracranial hypertension and impending uncal herniation refractory to profound sedation, osmotic agents and moderate hyperventilation. Decompressive craniectomy allowed an effective control of intracranial pressure and a favorable neurological outcome at discharge in both patients. Conclusions: Decompressive craniectomy could be considered as a rescue treatment strategy in patients with life-threatening intracranial hypertension due to severe herpetic encephalitis.

(Key words: Herpes, encephalitis, craniectomy, children).

Rev Chil Pediatr 2012; 83 (5): 468-473
\end{abstract}

\section{RESUMEN}

Introducción: La morbimortalidad de la encefalitis herpética ha disminuido con el uso de aciclovir. Sin embargo, algunos pacientes pueden evolucionar con necrosis focal hemorrágica y edema cerebral con posterior elevación de la presión intracraneal. Casos clínicos: Reportamos el curso clínico de dos niños con encefalitis herpética complicada con hipertensión intracraneal refractaria al tratamiento con sedación profunda, agentes osmóticos e hiperventilación. Ambos pacientes desarrollaron signos de enclavamiento uncal por lo que se realizó una craniectomía descompresiva precoz, que permitió un control de la presión intracraneal y una evolución neurológica favorable al alta. Conclusiones: La craniectomía descompresiva podría ser considerada como una alternativa terapéutica de rescate en pacientes con encefalitis herpética e hipertensión endocraneana refractaria a la terapia medica.

(Palabras clave: Herpes, encefalitis, craniectomía, niños).

Rev Chil Pediatr 2012; 83 (5): 468-473

Recibido el 6 de febrero de 2012, devuelto para corregir el 9 de mayo de 2012, segunda versión el 17 de junio de 2012, tercera versión el 4 de julio de 2012, aceptado para publicación el 28 de agosto de 2012.

Este trabajo cumple con los requisitos sobre consentimiento /asentimiento informado, comité de ética, financiamiento, estudios animales y sobre la ausencia de conflictos de intereses según corresponda.

Correspondencia a:

Raúl Bustos Betanzo

E-mail:rbustos@sanatorioaleman.cl 


\section{Introducción}

La encefalitis herpética (EH), es considerada como la causa más común de encefalitis esporádica fatal en pacientes de más de seis meses de edad en el mundo occidental ${ }^{1}$. La EH sin tratamiento tiene una mortalidad de $70 \%$ y menos del $3 \%$ de los pacientes son egresados libres de secuelas neurológicas. Después de la introducción del aciclovir, la mortalidad de la $\mathrm{EH}$ ha descendido a un $20 \%$ y la sobrevida podría aumentar si el tratamiento es iniciado dentro de cuatro días del debut de la enfermedad ${ }^{2}$. Sin embargo, algunos de estos pacientes van a evolucionar con hipertensión intracraneal (HTIC) severa secundaria a edema cerebral, hemorragia multifocal y necrosis cerebral.

Actualmente las guías para el tratamiento del trauma craneoencefálico (TCE) grave en niños, recomiendan, con distintos grados de evidencia, que la HTIC requiere de una terapia médica que de manera escalonada incluye sedo-analgesia, bloqueo neuromuscular, uso de agentes osmóticos, hiperventilación, barbitúricos e hipotermia ${ }^{3}$. Cuando la HTIC se hace refractaria al tratamiento médico se ha postulado el uso de la craniectomía descompresiva (CD) como terapia de rescate.

En nuestro país, existe sólo una publicación de esta técnica quirúrgica en un paciente adulto con $\mathrm{EH}$ grave y a nuestro entender no se han comunicado experiencias en casos pediátricos ${ }^{4}$.

El objetivo de este reporte es comunicar el uso de la $\mathrm{CD}$ en dos niños con EH complicada de edema cerebral e HTIC refractaria a tratamiento médico.

\section{Caso clínico 1}

Niño de 3 años, previamente sano, que fue ingresado a la unidad de cuidados intensivos (UCI) derivado desde otro hospital por fiebre de cuatro días de evolución, compromiso de conciencia y crisis convulsiva tónico clónica, por lo que se intubó previo a su traslado. Al examen de ingreso, destacó escala de Glasgow $7, \mathrm{~T}^{\circ} 39,2{ }^{\circ} \mathrm{C}$, actividad convulsiva tónico cló- nica generalizada. FC 112 p min, presión arterial $103 / 43 \mathrm{~mm} \mathrm{Hg}$. Los exámenes revelaron hemoglobina $8,3 \mathrm{~g} / \mathrm{dL}$, recuento de leucocitos $6480 \mathrm{~mm}^{3}$, plaquetas $137 \mathrm{mil} / \mathrm{uL}$, PCR 117 $\mathrm{mg} / \mathrm{L}$. líquido cefalorraquídeo (LCR) con 80 leucocitos $(90 \% \mathrm{PMN})$, ausencia de glóbulos rojos, proteínas 1,5 gL, glucosa $0,7 \mathrm{gL}$.

Se inició tratamiento con aciclovir y ceftriaxona. Se informó reacción de polimerasa en cadena (RPC) positiva para virus herpes 1 (VHS 1). El curso clínico del paciente se caracterizó por crisis convulsivas de difícil manejo requiriendo fenitoína, lorazepam, propofol. Un electroencefalograma demostró un proceso encefalopático generalizado con signos de irritabilidad cortical. Una tomografía computarizada (TC) cerebral reveló edema difuso. Se instaló un captor de presión intracraneal (PIC) de fibra óptica que demostró un valor de PIC en $24 \mathrm{mmHg}$ por más de $30 \mathrm{~min}$, iniciándose sedo-analgesia, bloqueo neuromuscular, administración de suero salino hipertónico al 3\% e hiperventilación moderada. La fiebre se trato con antipiréticos e hipotermia terapéutica a $34^{\circ} \mathrm{C}$ usando un sistema de enfriamiento externo (Blanketrol III). Todas las medidas anteriores permitieron una normalización de los valores de la PIC a menos de $15 \mathrm{mmHg}$.

A las $12 \mathrm{~h}$ de ingreso el paciente presentó un aumento persistente de la PIC hasta 38 $\mathrm{mmHg}$ y signos de enclavamiento uncal con midriasis derecha que no revirtieron con hiperventilación agresiva, suero salino hipertónico al 3\% y pentotal. La TC cerebral reveló aumento del edema cerebral e hipodensidad fronto parietal derecha, por lo que se realizó una $\mathrm{CD}$ fronto parieto temporal derecha y duroplastía de urgencia (figura 1a y 1b) lográndose una disminución y normalización mantenida de la PIC. La hipotermia controlada se mantuvo por 4 días con un recalentamiento posterior en $18 \mathrm{~h}$.

Un control del LCR demostró una RPC para VHS negativo. El paciente se trató 21 días con aciclovir, requirió 10 días en ventilación mecánica y 15 días en UCI. La Escala neurológica de Glasgow al egreso de la UCI fue 13. A los 8 meses post egreso hospitalario se realizó plastia craneal definitiva con malla de titanio y molde de acrílico, sin complicaciones. 


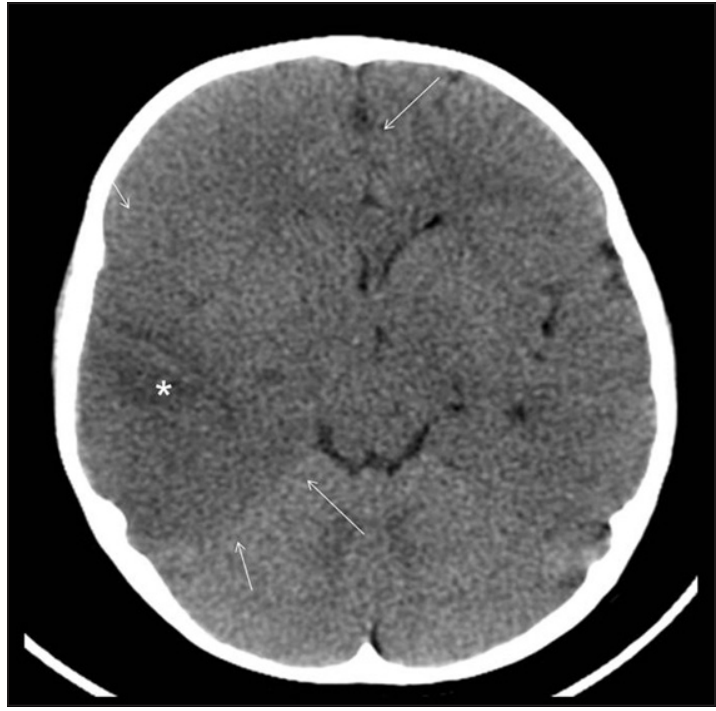

Figura 1a. TC cerebral sin contraste, efectuada al momento de la deterioración clínica del caso 1 muestra hipodensidad de la región frontal y temporo-parietal derecha, con efecto de masa sobre el ventrículo lateral de ese lado y desplazamiento de las estructuras de la línea media hacia la izquierda, compatible con edema cerebral.

A los 12 meses post alta hospitalaria la escala de discapacidad, según el Pediatric Cerebral Performance Category Score (PCPC) fue de 2, es decir el paciente se encontraba consciente, alerta, capaz de interactuar y con un trastorno neurológico menor ${ }^{5}$.

\section{Caso clínico 2}

Niña de un año, previamente sana, presentó un cuadro de 4 días de evolución de fiebre y coriza. Fue ingresada a otro hospital por 2 crisis tónico clónicas generalizadas, las que se repitieron en las $24 \mathrm{~h}$ siguientes a su hospitalización. Se realizó una TC de cerebro que se informó como normal y una PL que demostró un LCR con 22 leucocitos (91\% MN) y glóbulos rojos en regular cantidad. Desconocemos la razón de porque no se inició tratamiento con aciclovir o antibióticos. La paciente presentó una nueva convulsión tónico clónica acompañado de apnea por lo que es trasladada a nuestra UCI.

A su ingreso, la escala de Glasgow era de 7,

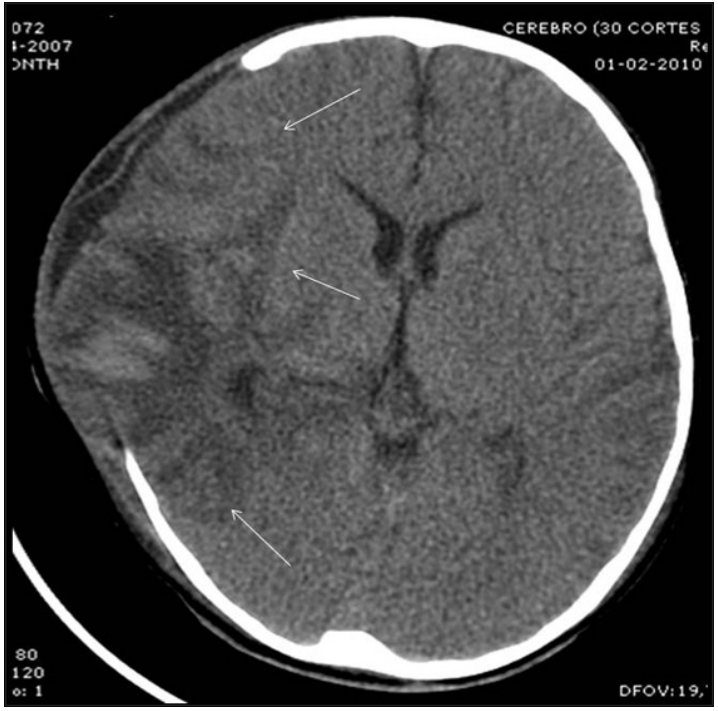

Figura 1b. TC cerebral efectuada después de la craniectomía descompresiva derecha en el caso 1, ya no muestra efecto de masa sobre las estructuras de la línea media y herniación lateral de los lóbulos parietal y temporal a través del defecto óseo.

por lo que se intubó y se conecto a ventilación mecánica. Una punción lumbar reveló un LCR con 31 leucocitos $(86 \% \mathrm{MN})$, proteínas $0,47 \mathrm{gL}$ y glucosa $0,59 \mathrm{gL}$. La TC de cerebro demostró una hipodensidad fronto temporal derecha con edema y desviación de la línea media.

Se inició tratamiento con sedo-analgesia, bloqueo neuromuscular, anticonvulsivantes, suero salino hipertónico, hiperventilación moderada y aciclovir asociado a ceftriaxona. El examen clínico demostró una midriasis arreactiva derecha por lo que se inició hiperventilación agresiva y pentotal, pese a lo cual los signos de enclavamiento persistieron. Finalmente, a las $2 \mathrm{~h}$ de ingreso se realizó una CD temporoparietal derecha y duroplastía. Se tomo biopsia cerebral.

La RPC del LCR se informó positiva para VHS 1 y el cultivo de LCR fue negativo. La biopsia cerebral demostró sustancia blanca con edema y abundantes células inflamatorias. La RPC del tejido cerebral también fue positiva para VHS 1.

Permaneció 8 días en la UCI, se trató 28 días con aciclovir por persistencia de PCR po- 
sitiva para VHS en el control de LCR al día 21 de evolución.

A los 6 meses post alta la paciente presenta una escala de discapacidad según el PCPC de 3 , lo que corresponde a un paciente consciente con una función por debajo de su edad y enfermedad neurológica no controlada que limita sus actividades. Se encuentra en tratamiento con anticonvulsivantes y en espera de la plastía craneal definitiva.

\section{Discusión}

A pesar de los beneficios demostrados de la monitorización y tratamiento de la HTIC en el TCE grave en niños, no existen guías clínicas en los casos de aumento de la PIC secundaria a encefalitis o meningitis. Se ha establecido que la HTIC es un hallazgo frecuente en las encefalitis graves de la edad pediátrica. Un 70\% de los casos de encefalitis se acompañarían de aumentos en la PIC, constituyéndose en un factor de mal pronóstico si se mantiene en el tiempo. La normalización de la PIC también podría mejorar la sobrevida en pacientes con meningitis bacteriana ${ }^{6,7}$. Es importante destacar que el control de la HTIC no sólo debe incluir el monitoreo de PIC, sino también parámetros clínicos y tomográficos para decidir la indicación y oportunidad de la $\mathrm{CD}$, ya que en ocasiones el registro de la PIC puede no reflejar en tiempo real lo que ocurre en la fosa temporal.

Si bien, el empleo de la CD sigue siendo controversial -aparece como evidencia de nivel III en las últimas guías de TCE- y existe un solo estudio randomizado de esta técnica en niños con TCE grave, su uso se ha extendido en la HTIC de origen no traumático refractaria al tratamiento médico .

El uso de la CD como tratamiento de rescate en infecciones del sistema nervioso central que cursan con HTIC y que no responden al tratamiento convencional, ha sido descrita en casos de empiema o encefalitis por Neisseria menigitidis, Listeria monocytogenes, Streptococcus pneumoniae, Mycoplasma pneumoniae y Toxoplasma gondii ${ }^{9-11}$.

En una revisión en Pubmed encontramos
6 casos pediátricos de $\mathrm{EH}$ que requirieron $\mathrm{CD}$ (tabla 1). La presentación clínica se caracterizó por fiebre y síndrome convulsivo. Todos eran previamente sanos a excepción de un sujeto, en tratamiento inmunosupresor. El VHS 1, aislado por RPC en LCR o biopsia cerebral fue el agente causal en todos los casos. En cuatro de los pacientes, incluido los aquí presentados, existió un inicio tardío del aciclovir desde el inicio de los síntomas. En la TC, la lesión fue de predominio derecho, encontrándose hipodensidad, edema e infartos cerebrales, desviación de línea media, borramiento de ventrículos y cisternas ${ }^{12-16}$. En todos los pacientes el enclavamiento inminente que motivó la $\mathrm{CD}$ ocurrió después de los cinco días del inicio de la enfermedad, hecho ya descrito en adultos ${ }^{17}$. Todos los pacientes sobrevivieron y las secuelas neurológicas observadas alcanzaron a más del 50\%. Cabe destacar que, en los tres casos en que el aciclovir fue iniciado precozmente en el curso de la enfermedad, esta fue capaz de progresar y llevar a aumentos en la PIC y secuelas neurológicas .

Una revisión reciente de 16 niños con EH destaca que, a pesar de que todos los pacientes de esta serie fueron tratados con aciclovir dentro de los tres primeros días de la enfermedad, el $63 \%$ de los pacientes presentaron una secuela neurológica al alta ${ }^{18}$.

El uso de la hipotermia controlada se ha mostrado eficaz en la reducción de la PIC en el edema cerebral secundario al TCE grave y en la falla hepática aguda. En menigo-encefalitis la hipotermia no ha sido objeto de estudios rigurosos. En el paciente en que la utilizamos, esta técnica no permitió un control efectivo de la PIC, lo que contrasta con reportes en pacientes adultos con $\mathrm{EH}$ en el que uso de hipotermia prolongada, usando sistemas de enfriamiento intravascular, han permitido un control rápido y permanente de la PIC, evitándose medidas más agresivas como la $\mathrm{CD}^{19,20}$.

Creemos que una $\mathrm{CD}$ realizada precozmente debería ser considerada en aquellos pacientes pediátricos con HTIC de causa no traumática -como es el caso de la EH grave- que presenten mala repuesta al tratamiento médico para así evitar secuelas neurológicas graves o un desenlace fatal. 
Tabla 1. Características de los pacientes pediátricos reportados en la literatura sometidos a craniectomía descompresiva por encefalitis herpética

\begin{tabular}{|c|c|c|c|c|c|c|c|}
\hline $\begin{array}{l}\text { Autor } \\
\text { Año } \\
\text { Referencia }\end{array}$ & $\begin{array}{l}\text { Edad/ } \\
\text { sexo }\end{array}$ & $\begin{array}{l}\text { Hallazgos } \\
\text { clínicos }\end{array}$ & $\begin{array}{l}\text { Tipo VHS en } \\
\text { RPC de LCR o } \\
\text { Bx cerebral }\end{array}$ & $\begin{array}{l}\text { Latencia } \\
\text { síntomas } \\
\text { e inicio de } \\
\text { aciclovir } \\
\text { días }\end{array}$ & $\begin{array}{l}\text { Hallazgos } \\
\text { en TC }\end{array}$ & $\begin{array}{c}\text { Craniectomía } \\
\text { descompresiva } \\
\text { días de } \\
\text { evolución }\end{array}$ & $\begin{array}{l}\text { Desenlace } \\
\text { y secuelas }\end{array}$ \\
\hline $\begin{array}{l}\text { Ebel } \\
1999 \\
(12)\end{array}$ & $8 \mathrm{~m} / \mathrm{f}$ & $\begin{array}{l}\text { Somnolencia } \\
\text { CTC }\end{array}$ & LCR/VHS-1 & 2 & $\begin{array}{l}\text { Hemorragia } \\
\text { TP izquierda } \\
\text { Edema }\end{array}$ & 5 & $\begin{array}{l}\text { Vivo } \\
\text { Paresia facial }\end{array}$ \\
\hline $\begin{array}{l}\text { Kannu } \\
2004 \\
(13)\end{array}$ & $9 \mathrm{a} / \mathrm{f}$ & $\begin{array}{l}\text { Fiebre } \\
\text { CTC }\end{array}$ & LCR/VHS-1 & 3 & $\begin{array}{l}\text { Edema } \\
\text { Hiperdens } \\
\text { P derecha }\end{array}$ & 10 & $\begin{array}{l}\text { Vivo } \\
\text { Afasia } \\
\text { epilepsia }\end{array}$ \\
\hline $\begin{array}{l}\text { Bayram } \\
2008 \\
(14)\end{array}$ & $15 \mathrm{a} / \mathrm{f}$ & $\begin{array}{l}\text { Fiebre, convulsión } \\
\text { focal }\end{array}$ & LCR/VHS-1 & 7 & $\begin{array}{l}\text { Edema } \\
\text { Hipodens } \\
\text { TP derecha }\end{array}$ & 13 & $\begin{array}{l}\text { Vivo } \\
\text { Sin secuelas }\end{array}$ \\
\hline \multirow[t]{2}{*}{$\begin{array}{l}\text { González } \\
2008 \\
(15)\end{array}$} & $13 \mathrm{a} / \mathrm{m}$ & Convulsión focal & VHS-1 & 2 & $\begin{array}{l}\text { Hipodensidad } \\
\text { T derecha }\end{array}$ & 14 & $\begin{array}{l}\text { Vivo } \\
\text { Sin secuelas }\end{array}$ \\
\hline & $16 \mathrm{a} / \mathrm{f}$ & $\begin{array}{l}\text { Cefalea } \\
\text { hemiparesia }\end{array}$ & VHS-1 & 7 & $\begin{array}{l}\text { Hipodensidad } \\
\text { FT derecha }\end{array}$ & 12 & $\begin{array}{l}\text { Vivo } \\
\text { Sin secuelas }\end{array}$ \\
\hline $\begin{array}{l}\text { Sánchez } \\
2008 \\
(16)\end{array}$ & $6 \mathrm{a} / \mathrm{f}$ & $\begin{array}{l}\text { Fiebre } \\
\text { Status convulsivo }\end{array}$ & $\begin{array}{l}\text { Bx cerebral / } \\
\text { VHS-1 }\end{array}$ & 7 & $\begin{array}{l}\text { Lesiones } \\
\text { hemorrágicas T } \\
\text { derecha edema }\end{array}$ & 10 & $\begin{array}{l}\text { Vivo } \\
\text { Deficit } \\
\text { cognitivo } \\
\text { menor }\end{array}$ \\
\hline \multirow[t]{2}{*}{$\begin{array}{l}\text { Casos } \\
\text { presentados }\end{array}$} & $3 \mathrm{a} / \mathrm{m}$ & $\begin{array}{l}\text { Fiebre } \\
\text { Status convulsivo }\end{array}$ & LCR VHS -1 & 4 & $\begin{array}{l}\text { Edema } \\
\text { Hipodens TP } \\
\text { derecha }\end{array}$ & 2 & $\begin{array}{l}\text { Vivo } \\
\text { Déficit } \\
\text { menor }\end{array}$ \\
\hline & $1 \mathrm{a} / \mathrm{f}$ & $\begin{array}{l}\text { Fiebre } \\
\text { Convulsión focal }\end{array}$ & $\begin{array}{l}\text { LCR VHS-1 y Bx } \\
\text { cerebral }\end{array}$ & 7 & $\begin{array}{l}\text { Hipodens FT } \\
\text { der }\end{array}$ & 7 & $\begin{array}{l}\text { Vivo } \\
\text { Epilpesia }\end{array}$ \\
\hline
\end{tabular}

VHS: virus herpes simplex. Bx: biopsia. LCR: líquido céfalo raquídeo. CTC: convulsión tónico clónica. TP: temporo parietal FT: fronto temporal. T: temporal.

\section{Referencias}

1.- Whitley RJ, Kimberling DW: Herpes Simplex Encephalitis: Children and Adolescents. Semin Pediatr Infect Dis. 2005; 16: 17-23

2.- Whitley RJ, Gnann JW: Viral encephalitis: familiar infections and emerging pathogens. Lancet 2002; 359: 507-13.

3.- Adelson PD, Bratton SL, Carney NA, et al: Guidelines for the acute medical management of severe traumatic brain injury in infants, children and adolescents. Second edition. Pediatr Crit Care Med 2012; 13: S1-S75.

4.- Mellado P, Castillo L, Andresen $M$, Campos $M$, $P$ érez $C$, B audrand $R$ : Decompressive craniectomy in a patient with herpetic encephalitis associated to refractory intracranial hypertension. Rev Med Chile 2003; 131: 1434-8.

5.- Fiser D: Assessing the outcome of pediatric intensive care. J Pediatr 1992; 121: 68-74.
6.- Rebaud P, Berthier JC, Hartemann E, Floret D: Intracranial pressure in childhood central nervous system infections. Intensive Care Med 1988; 14: 522-5.

7.- Lindvall P, Ahlm C, Ericsson M, Gothefors L, Naredi S, Koskinen D: Reducing intracranial pressure may increase survival among patients with bacterial meningitis. Clin Infect Dis 2004; 38: 384-90.

8.- Taylor A, Butt W, Rosenfeld J, et al: A randomized trial of very early decompressive craniectomy in children with traumatic brain injury and sustained intracranial hypertension. Child's Nerv Syst 2001; 17: 154-62.

9.- Aghakhani N, Durán P, Chevret L, et al: Decompressive craniectomy in children with non traumatic refractory high intracranial pressure. Clinical article. J Neurosurg Pediatr 2009; 3: 66-9.

10.- Agrawal D, Hussain N: Decompressive craniectomy in cerebral toxoplasmosis. Eur J Clin Microbiol Infect Dis 2005; 24: 772-3.

11.- Perin P, Nascimben E, Longatti P: Decompressive cra- 
niectomy in a case of intractable intracranial hypertension due to pneumococcal meningitis. Acta Neurocchir (Wien) 2008; 150: 837-42.

12.- Ebel H, Kutcha J, Baloch A, Klug N: Operative treatment of tentorial herniation in herpes encephalitis. Child's Nerv Syst 1999; 15: 84-6.

13.- Kannu P, Pinnock R: Uncommon complication of herpes simplex encephalitis.J Paediatr Child Health 2004; 40: 711-3.

14.- Bayram N, Ciftdogan DY, Karapinar B, et al: A case of herpes simplex encephalitis revealed by decompressivecraniectomy. Eur J Pediatr 2008; 167: 821-2.

15.- González-Rabelino GA, Fons C, Rey A, Roussos I, Campistol J: Craniectomy in herpetic encephalitis. Pediatr Neurol. 2008; 39: 201-3.

16.- Sánchez-Carpintero R, Aguilera S, Idoate M, Bejarano
$B$ : Temporal lobectomy in acute complicated herpes simplex encephalitis: technical case report. Neurosurgery 2008; 62: E1174-5.

17.- Barnett GH, Ropper AH, Romeo J: Intracranial pressure and outcome in adult encephalitis. J Neurosurg 1998; 68: 585-8.

18.- Elbers JM, Bitnun A, Richardson SE, et al: A 12-Year Prospective Study of Childhood Herpes Simplex Encephalitis: Is There a Broader Spectrum of Disease?. Pediatrics 2007; 119: e399-e407.

19.- Kutleša M, Baršić B, Lepur D: Therapeutic hypothermia for adult viral meningoencephalitis. Neurocrit Care. 2011; 15: 151-5.

20.- Wagner I, Staykov D, Volbers B, Kloska S: Therapeutic hypothermia for space occupying herpes simplex virus encephalitis Minerva Anestesiol. 2011; 77: 371 -4 